Annales Geophysicae (2003) 21: 933-945 (C) European Geosciences Union 2003

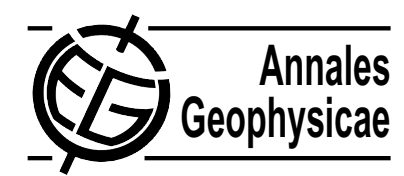

\title{
Optical signatures of auroral arcs produced by field line resonances: comparison with satellite observations and modeling
}

\author{
J. C. Samson ${ }^{1}$, R. Rankin ${ }^{3}$, and V. T. Tikhonchuk ${ }^{1, ~ *}$ \\ ${ }^{1}$ Physics Department, University of Alberta, Edmonton, Alberta, Canada \\ ${ }^{2}$ Institute of Fundamental Physics, University of Bordeaux 1, Talence 33405, France \\ * on leave from P. N. Lebedev Physics Institute, Russian Academy of Science, Moscow, Russia
}

Received: 13 December 2001 - Revised: 21 October 2002 - Accepted: 23 October 2002

\begin{abstract}
We show two examples from the CANOPUS array of the optical signatures of auroral arcs produced by field line resonances on the night of 31 January 1997. The first example occurs during local evening at about 18:00 MLT (Magnetic Local Time), where CANOPUS meridian scanning photometer data show all the classic features of field line resonances. There are two, near-monochromatic resonances (at approximately 2.0 and $2.5 \mathrm{mHz}$ ) and both show latitudinal peaks in amplitude with an approximately 180 degree latitudinal phase shift across the maximum. The second field line resonance event occurs closer to local midnight, between approximately 22:00 and 22:40 MLT. Magnetometer and optical data show that the field line resonance has a very low frequency, near $1.3 \mathrm{mHz}$. All-sky imager data from CANOPUS show that in this event the field line resonances produce auroral arcs with westward propagation, with arc widths of about $10 \mathrm{~km}$. Electron energies are on the order of $1 \mathrm{keV}$. This event was also seen in data from the FAST satellite (Lotko et al., 1998), and we compare our observations with those of Lotko et al. (1998). A remarkable feature of this field line resonance is that the latitudinal phase shift was substantially greater than 180 degrees. In our discussion, we present a model of field line resonances which accounts for the dominant physical effects and which is in good agreement with the observations. We emphasize three points. First, the low frequency of the field line resonance in the second event is likely due to the stretched topology of the magnetotail field lines, with the field line resonance on field lines threading the earthward edge of the plasma sheet. Second, the latitudinal phase structure may indicate dispersive effects due to electron trapping or finite ion gyroradius. Third, we show that a nonlocal conductivity model can easily explain the parallel electric fields and the precipitating electron energies seen in the field line resonance.
\end{abstract}

Key words. Magnetospheric physics (electric fields; energetic particles precipitating; current systems)

Correspondence to: J. C. Samson

(samson@space.ualberta.ca)

\section{Introduction}

Numerous ground-based observations have shown that ultralow frequency field line resonances (FLRs) in the $1-4 \mathrm{mHz}$ band influence the formation of some auroral arcs. Recent FAST satellite (Carlson et al., 1998) observations (Lotko et al., 1998) have now confirmed that these FLRs are associated with particle acceleration processes on field lines threading the auroral ionosphere. Lotko et al. (1998) attributed the formation of parallel electric fields in a $1.3 \mathrm{mHz}$ FLR to dispersive effects in the Alfvénic structures and regions of anomalous resistivity in the large field-aligned currents associated with the FLR. In this paper, we take a detailed look at this event as seen by the CANOPUS (Rostoker et al., 1994) magnetometers, meridian scanning photometer array (MPA), and all-sky imager (ASI). We shall address the reasons for the very low frequency of the FLR, an issue that caused Lotko et al. (1998) some concern, and also discuss a plausible mechanism for the formation of field-aligned electric fields through a nonlocal electron kinetic response. These nonlocal effects, for which we will use the term nonlocal conductivity, include a consideration of precipitating electrons, and mirroring of electrons along the magnetic field line. We shall show that this mechanism gives a very plausible explanation for the parallel electric fields seen in the FLR, without the need for anomalous resistivity.

The mechanisms leading to the auroral arc can be grouped in two areas, the generator and the accelerator. The generator is the source of free energy (usually in the magnetosphere) that produces the field-aligned currents, and the magnetic and electric fields associated with auroral arcs. Some possible generators include shear flow in magnetospheric convection, pressure gradients at small angles to gradients in flux tube volume (Lyons and Samson, 1992), reconnection and X-line formation (Atkinson, 1992) and compressional MHD energy that excites FLRs (Samson et al., 1996). Borovsky (1993) discusses a variety of the mechanisms, excluding the FLR. Most evidence now indicates that the accelerator giving the precipitating auroral electrons is due to a parallel elec- 


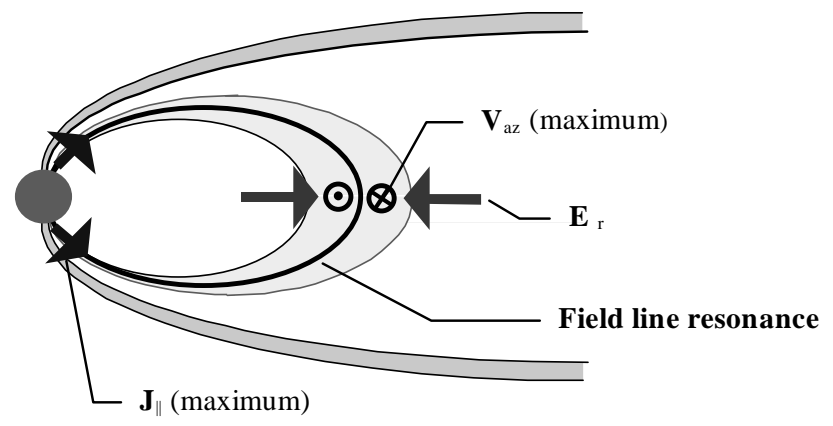

Fig. 1. A schematic of an FLR in the near-Earth magnetotail. The outer shaded region is the plasma sheet boundary layer. The inner shaded region is the earthward edge of the plasma sheet, where precipitating energetic hydrogen ions (10 $\mathrm{s}$ of $\mathrm{keV}$ ) give the $\mathrm{H}-\beta$ band seen in the MPA data.

tric field, and much experimental and theoretical work has concentrated on mechanisms to produce such a parallel electric field. Examples include static magnetosphere-ionosphere coupling, including mirroring of electrons (Chiu and Schulz, 1978; Knight, 1973), double layers, and dispersion in kinetic or electron inertia Alfvén waves (Borovsky, 1993).

The FLR model gives a relatively simple and selfconsistent explanation for a number of spatial scales seen in auroral arcs, and further clues to the accelerator mechanism, when kinetic effects for electrons are considered. The source of energy for monochromatic, ULF FLRs is believed to be compressional, near-monochromatic fast MHD waves formed in magnetospheric waveguides or cavities (Samson et al., 1992a; Liu et al., 1994). This is still a very active area of study, and results are not conclusive. The compressional energy couples to the shear Alfvén wave at the resonance position defined by $\omega^{2}-V_{A}^{2} k_{\|}^{2}=0$, where $V_{A}$ is the local Alfvén speed. A schematic for a FLR in the near-Earth magnetotail is given in Fig. 1, showing a FLR threading the inner plasma sheet (shaded region). The resonance shown here is the fundamental shear Alfvén mode standing between the highly conducting auroral ionospheres. Typical frequencies of the resonances measured from the ground are in the range of $1-4 \mathrm{mHz}$.

According to ground-based observations and models (Samson et al., 1996), the maximum in the field-aligned current (FAC), $J_{\|}$, is 90 degrees out of phase with the maximum in the transferse electric field, $E_{x}$. Here, and in the rest of the text, we will use subscript $x$ to denote the component perpendicular to the magnetic shell (radial in the equatorial plane), subscript $y$ for the azimuthal component, and $z$ for the parallel or field-aligned component. Maximum $J_{\|}$above the ionosphere is in the range of many $\mu \mathrm{A} / \mathrm{m}^{2}$. The maximum in the azimuthal velocity field in the equatorial plane can be of the order of $100 \mathrm{~s}$ of $\mathrm{km} / \mathrm{s}$ (Mitchell et al., 1990). The net perpendicular potential change in the equatorial plane can be of the order of several $\mathrm{keV}$. The azimuthal wavelength of the FLR can be $10 \mathrm{~s}$ to $100 \mathrm{~s}$ of degrees, and typical azimuthal propagation speeds are on the order of 1 to 10 degrees/s. Near the

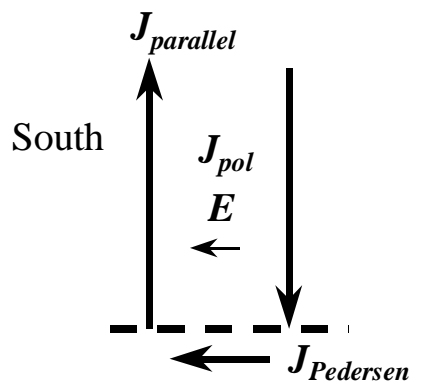

Phase 1

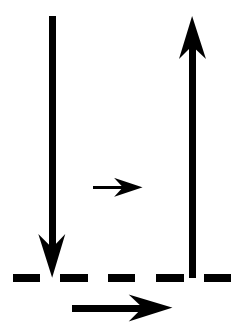

Phase 3

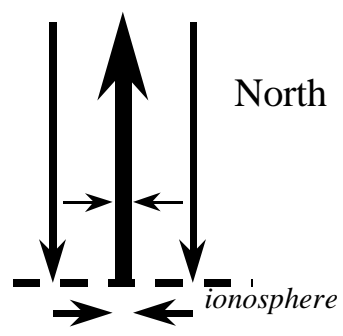

Phase 2
Fig. 2. A schematic of currents and fields in field line resonances near the ionosphere, shown in phases 90 degrees apart.

auroral ionosphere, the latitudinal thickness of the FLR is of the order of $10 \mathrm{~s}$ of kilometers, and the thickness of the upward field-aligned current region, which shows temporally periodic restructuring, is on the order of $10 \mathrm{~km}$. Figure 2 gives a schematic showing four phases of the FAC and wave fields of a FLR near the ionosphere. We shall discuss this schematic in more detail when looking at the experimental data and simulations, but a few comments are in order. If we correlate upward FAC with auroral luminosity, then it becomes clear that in certain phases, as the FLR propagates azimuthally, two parallel arcs can exist, separated by the approximate width of the FLR (Phase 4). In the ASI data from CANOPUS, we label the poleward arc as arc-1, and the equatorward arc as arc-2. At other times, one brighter arc will be seen (Phase 2). Meridian scanning photometer data will show luminosity bands moving periodically poleward (note the poleward motion of the upward current in the 4 phases in Fig. 2).

The FLR mechanism can explain the observed amplitudes of the transverse ( $x$-component) electric field, the fieldaligned current, and the observed latitudinal scales in auroral arcs, if one takes into account both nonlinear and dispersive effects in FLRs (Rankin et al., 1999a). Nonlinear ponderomotive forces in the FLR will produce density cavities with the same latitudinal scale size as the FLR, i.e. $10 \mathrm{~s}$ of $\mathrm{km}$ in latitude above the auroral ionosphere (Rankin et al., 1999b). The model presented in Rankin et al. (1999a, b) also predicts double arc structures during part of the FLR cycle, separated 


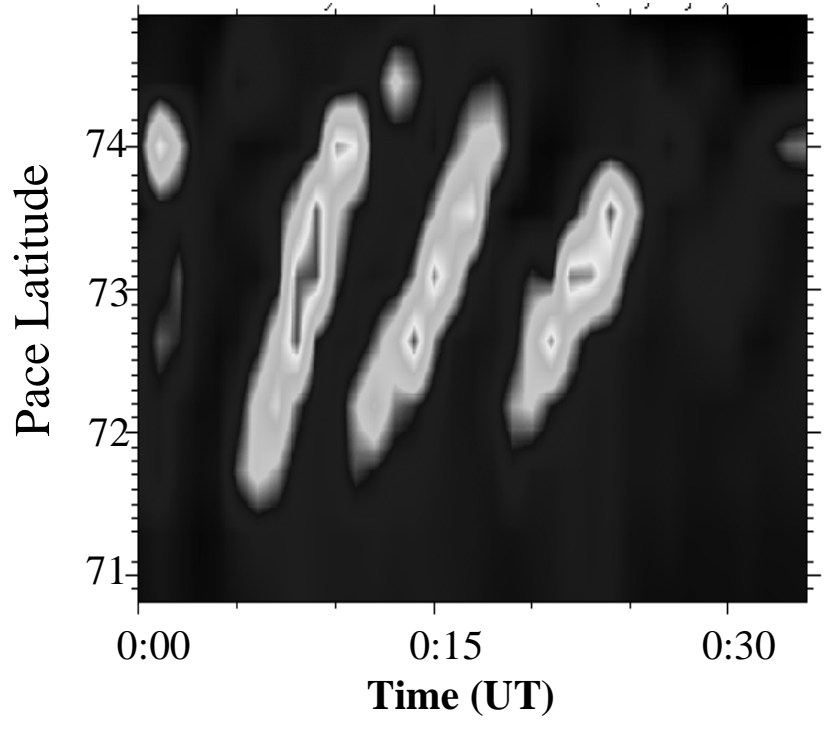

Fig. 3. Field line resonances seen in the $557.7 \mathrm{~nm}$ emissions from the MSP at Rankin Inlet on 31 January 1997, $4 \mathrm{~h}$ before the FAST pass. These data use the standard latitudinal binning, with a latitudinal resolution of $\sim 0.5$ degrees. The coordinates used here are PACE (Ruohoniemi et al., 1991). EDFL coordinates are approximately PACE -3 degrees. The grey scale ranges from 0 Rayleighs (black) to 2000 Rayleighs (white) to 4000 Rayleighs (black again).

by the latitudinal width of the FLR, or $10 \mathrm{~s}$ of $\mathrm{km}$. Individual discrete arcs will have a width of about $10 \mathrm{~km}$, based on the width of the upward FAC in the FLR. A smaller, subkilometer scale structure in the arc could be associated with a filamentation instability in the FAC. However, this process, as well as two other nonlinear mechanisms in the FLR which could be responsible for small-scale structuring, namely tearing in the large FAC above the auroral ionosphere, and shear flow instabilities in the equatorial plane of the FLR, will not be considered here.

In this paper, we will first review CANOPUS observations, including magnetometer and optical data, for two typical FLR events on 31 January 1997. The first event at 18:00 MLT shows classical FLR features with many important details. The second event occurred the same day, but $4 \mathrm{~h}$ later. It will be compared with FAST satellite observations in the interval where the satellite traverses the $1.3 \mathrm{mHz}$ FLR at approximately 04:25 to 04:26 UT on 31 January at an altitude of $4146 \mathrm{~km}$ (Lotko et al., 1998). Following these observations, we consider a model of FLR eigenmodes that accounts for their excitation, nonlinear evolution, and formation in the acceleration zone above the ionosphere. We show that the FLR mode on stretched field lines has the same low frequency as seen in the CANOPUS data. We then consider mechanisms for the formation of parallel electric fields in the FLR, and conclude that kinetic effects, including trapped and precipitating electrons, give the most likely mechanism to produce the observed electron energies and parallel electric fields.

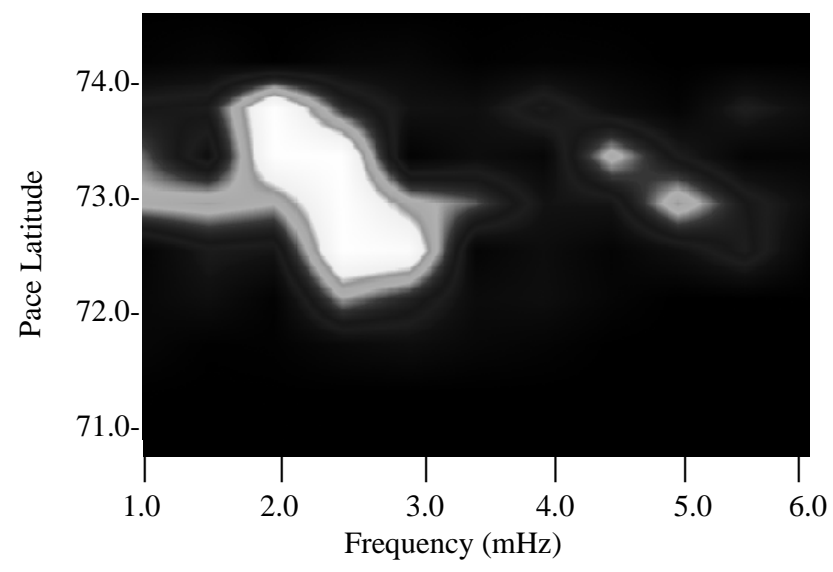

Fig. 4. Latitude dependent power spectra for the optical data in Fig. 3. Power is in arbitrary units, with white indicating the largest powers.

\section{Observations of the night-side FLRs events}

We use magnetometer data from three CANOPUS stations: Fort Churchill (FCHU, EDFL 66.3 N, 336.7 E), Gillam (GILL, 63.9 N, 336.2 E), and Island Lake (ISLL, $61.4 \mathrm{~N}$, $336.4 \mathrm{E}), 630.0 \mathrm{~nm}$ data from the ASI at GILL, $557.7 \mathrm{~nm}$ meridian photometer array (MPA) data from Rankin Inlet and 486.1 and $630.0 \mathrm{~nm}$ high resolution MPA data from GILL. The coordinates used in presenting the data for the FAST event are eccentric dipole (EDFL), in order to be compatible with the coordinates used in Lotko et al. (1998). The instruments in CANOPUS are discussed in detail by Rostoker et al. (1994). Near zenith, the ASI has a resolution of about $1-2 \mathrm{~km}$, depending on the height of the $630.0 \mathrm{~nm}$ emissions. The resolution of the high resolution MPA data at zenith is on the order of 0.5 to $1 \mathrm{~km}$, depending on the height of the emissions. The magnetometer data are recorded in geodetic coordinates ( $x$ - north, $y$ - east, $z$-downward), but the declination at these three stations is less than 3 degrees, and consequently, the $x$-component is essentially equivalent to magnetic north.

To facilitate comparison of the optical signatures of FLRs with those seen in radar data, we first show a multiple resonance structure that has a very broad latitudinal width. Meridian scanning optical data are shown in Fig. 3 for the first event at 18:00 LT (Local Time). The poleward moving bands of optical emissions can easily be compared with the poleward moving bands in the Doppler velocity data for FLRs seen by HF-radars (see Ruohoniemi et al., 1991, Plate 2; Fenrich et al., 1995, Fig. 2). We show later, in a second example, that these poleward moving bands are associated with auroral arcs.

This example illustrates a number of important points for the interpretation of the optical signatures of FLRs. First, multiple discrete resonances can occur at closely spaced latitudes. Though not clear in the plot of the latitudinal power spectra shown in Fig. 4, two peaks exist, one at $2.5 \mathrm{mHz}$, with 

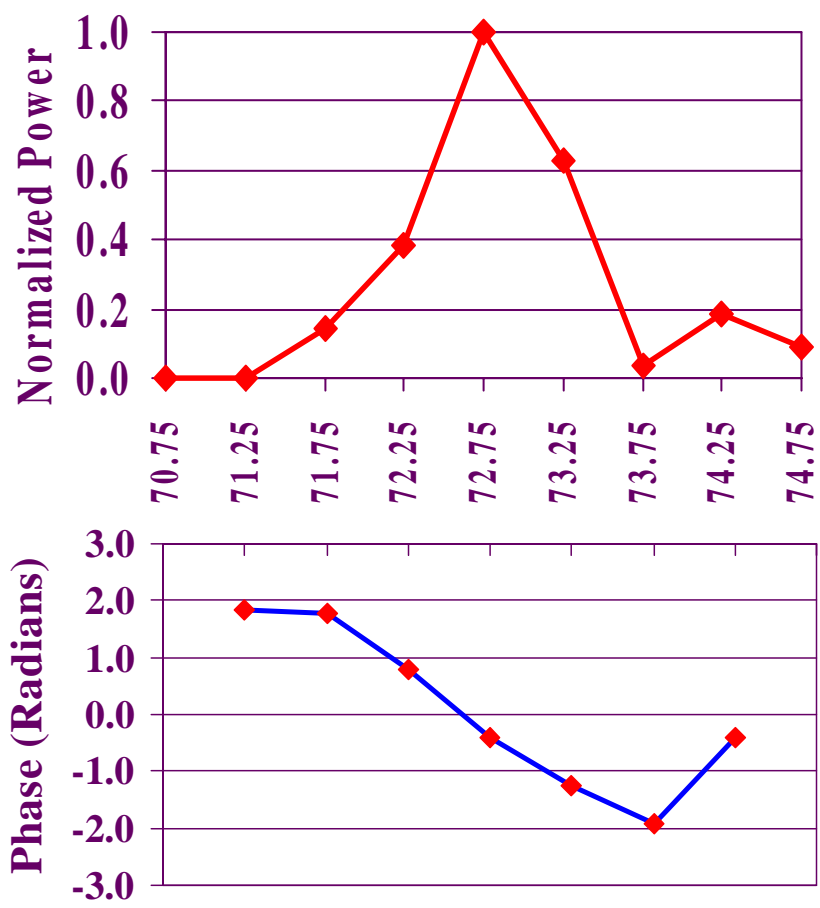

\section{Latitude}

Fig. 5. Latitude dependent power and phase for the $2.5 \mathrm{mHz}$ FLR seen in Fig. 3. The power is the square of the Fourier amplitude, and the phase is estimated from the ratio of the imaginary and real Fourier component at $2.5 \mathrm{mHz}$.

a maximum near $72.5^{\circ}-73.0^{\circ}$ and one at $2 \mathrm{mHz}$ with a maximum at approximately $73.5^{\circ}$. Multiple spectral peaks were also seen in the data presented by Ruohoniemi et al. (1991). The two peaks are most clearly seen in the harmonics at approximately 4 and $5 \mathrm{mHz}$. Note that these harmonics are not generated on FLRs in the magnetosphere, but are most likely due to harmonic structure produced by the electron precipitation and the associated production of optical emissions. For FLR harmonics within the magnetospheric plasma, the frequencies are not integer multiples of the fundamental (contrary to what is seen in Fig. 4). The harmonics are generated in the optical data because precipitation, and the production of optical luminosity, is not a linear function of $j_{\|}$. The sequence with lower frequencies at higher latitudes is a characteristic signature of FLRs.

A second feature of importance is the latitudinal phase shift across the maximum amplitude of the FLR. Figure 5 illustrates that the $2.5 \mathrm{mHz}$ FLR shows the "classic" $180^{\circ}$ phase decrease with increasing latitude. If there is not sufficient spectral resolution, then the mixture of the 2 and $2.5 \mathrm{mHz}$ signatures would possibly lead to erroneous estimates with phase shifts larger than $180^{\circ}$. This point will be important in the analysis of our second event, as the phase shift for this FLR is much greater than $180^{\circ}$. For the second event, however, we have established that there is only a single resonance at $1.3 \mathrm{mHz}$ and consequently, the larger latitudinal phase shift must be attributed to another mechanism.

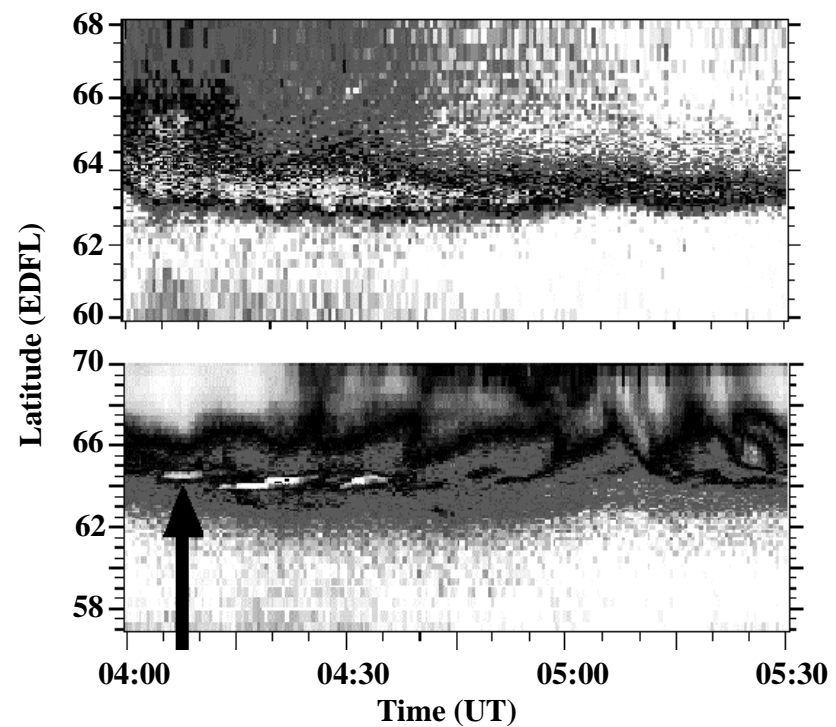

Fig. 6. Optical emissions at $486.1 \mathrm{~nm}$ (top) and $630.0 \mathrm{~nm}$ (bottom) from the high resolution MPA data from GILL. The grey scale for the $486.1 \mathrm{~nm}$ emissions ranges from zero $\mathrm{R}$ (white) to $15 \mathrm{R}$ (black) to $30 \mathrm{R}$ (white again). The maximum in the $486.1 \mathrm{~nm}$ emissions is evident in the white band between 63 and 64 degrees latitude: the range of the $630.0 \mathrm{~nm}$ emissions is 0 to $1000 \mathrm{R}$. The FLRs (indicated by the arrow) are seen as white poleward moving bands (indicating a maximum in emissions of about $1000 \mathrm{R}$ ).

MPA data of the second event on 31 January 1997 are shown in Fig. 6, magnetometer data in Fig. 7, and ASI data in Fig. 8. The power spectrum in Fig. 7 indicates a possible FLR with a frequency of about $1.3 \mathrm{mHz}$. Fields associated with this FLR were recorded by the FAST satellite. Passing through the $1.3 \mathrm{mHz}$ FLR at an altitude of $4146 \mathrm{~km}$ and a speed of $5 \mathrm{~km} / \mathrm{s}$, the FAST data show only a quick "snapshot" of the fields and currents associated with one phase of the FLR (Lotko et al., 1998). The latitudinal extent of fields associated with the FLR at FAST altitudes is approximately $150 \mathrm{~km}$, mapping to approximately $70 \mathrm{~km}$ in the auroral ionosphere. Downward FAC currents are seen on the equatorward side of the FLR, and upward currents on the poleward side, corresponding to Phase 3 in the schematic in Fig. 2. Magnitudes are on the order of $2-3 \mu \mathrm{A} / \mathrm{m}^{2}$. The upward FAC is associated with a downward electron flux of field-aligned, suprathermal electrons. The electron energies range from $1 \mathrm{keV}$ on the poleward edge, to less than $300 \mathrm{eV}$ on the equatorward edge of the arc, which is $20 \mathrm{~km}$ thick at the FAST altitude (mapping to approximately $10 \mathrm{~km}$ at the ionosphere). The downward electron energy flux of $1.6 \mathrm{~mW} / \mathrm{m}^{2}$ is adequate to produce the auroral arc seen in the $630.0 \mathrm{~nm}$ data from the CANOPUS ASI at Gillam (Fig. 6). Line intensity ratio measurements (630.0/557.0) from the ASI indicate a precipitating electron energy of slightly greater than $1 \mathrm{keV}$. FAST data show that perpendicular electric fields had maxima on the order of $150-180 \mathrm{mV} / \mathrm{m}$.

We attribute the low frequency of the FLR seen by FAST to the stretching of tailward magnetic field lines. Evidence 

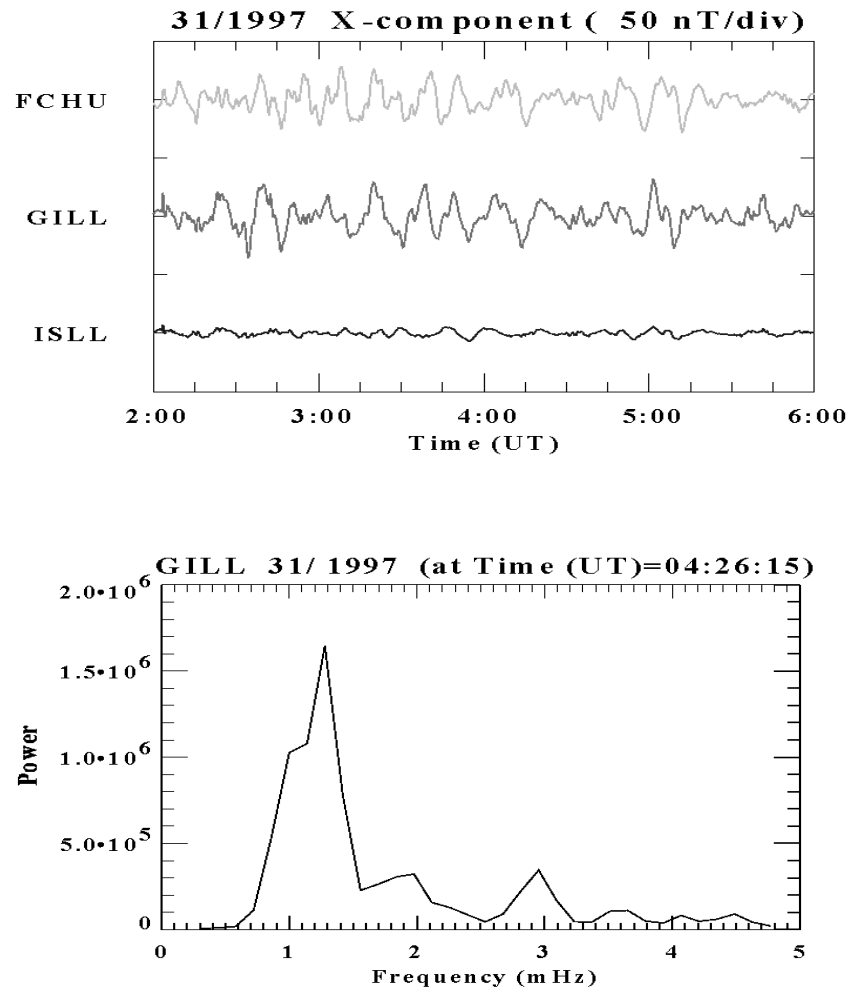

Fig. 7. High passed $(0.5 \mathrm{mHz})$ magnetometer data and the Fourier power spectrum for the data at GILL.

for this is seen from the ground as diffuse optical H- $\beta$, produced by energetic ions that undergo pitch-angle scattering near the earthward edge of the plasma sheet. Numerous studies have in the past shown that the region of energetic ion precipitation is on stretched field lines (Sergeev et al., 1993; Newell et al., 1998). The 630.0 and $486.1 \mathrm{~nm}(\mathrm{H}-\beta)$ MPA data for the FAST event are shown in Fig. 6. The 13-min, periodic, poleward moving bands associated with the FLR are very clear in the $630.0 \mathrm{~nm}$ data, and are pointed out by an arrow indicating the FLR. On comparing the $630.0 \mathrm{~nm}$ data with the $486.1 \mathrm{~nm}$ data, it can be seen that the FLR is located on the poleward border of a region of energetic ( $10 \mathrm{~s}$ of $\mathrm{keV})$ hydrogen ion precipitation (Samson et al., 1992b). Consequently, the poleward border of the $\mathrm{H}-\beta$, where the FLR is found, should have a stretched topology. The field radius of curvature near the equatorial plane, $R_{c}$, should be comparable to the gyroradius of energetic hydrogen ions $\rho_{i}$, allowing nonadiabatic scattering and isotropization of the hydrogen ion distribution functions, leading to enhanced precipitation. For $10 \mathrm{keV}$ protons in a magnetic field of $10 \mathrm{nT}$, the gyroradius is about $1000 \mathrm{~km}$. This gives the approximate radius of curvature for the magnetic field line. It is an order of magnitude smaller than the radius of curvature for the corresponding dipolar line. Earthward of this region, where the magnetic field topology is more dipolar, the ions become trapped, and no $\mathrm{H}-\beta$ emissions are seen. This stretched topology, associated with pressure gradients near the earthward edge of the plasma sheet and the outer edge of the ring current, can

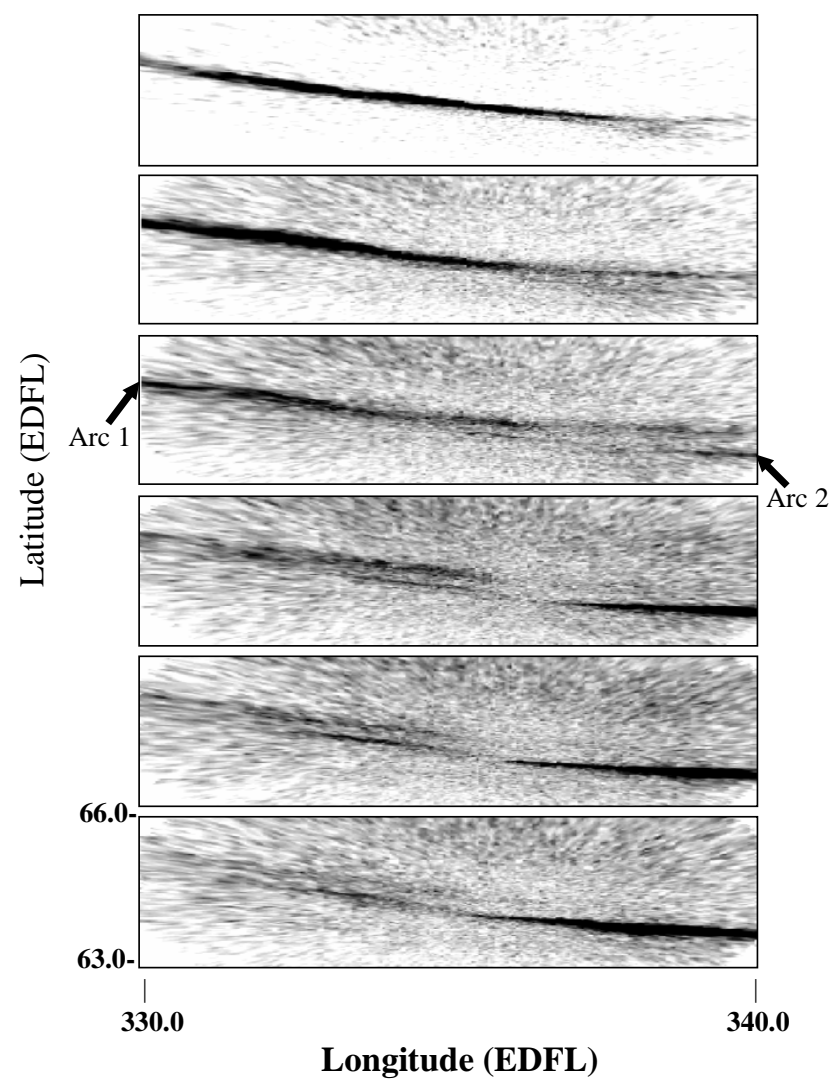

Fig. 8. ASI images of the $630.0 \mathrm{~nm}$ auroral arc associated with the $1.3 \mathrm{mHz}$ FLR. The grey scale ranges from 0 (white) to $600 \mathrm{R}$ (black). The times of the images (top to bottom) are 04:22:03, 04:24:03, 04:26:03, 04:28:03, 04:29:03, 04:30:03 UT.

substantially reduce the frequency of the FLRs (Chan et al., 1994; Rankin et al., 2000) when compared to the frequencies that might be expected from a more dipolar topology.

Another interesting observation is the 13-min, quasiperiodic undulations of the equatorward cutoff of the more diffuse $630.0 \mathrm{~nm}$ emissions. This cutoff marks the field lines mapping to the inner edge of the electron plasma sheet (Samson, 1994). These undulations may indicate that the FLR was driven by a magnetotail cavity mode (Liu et al., 1994), not the flank MHD waveguide postulated by Samson et al. (1992a). The apparent westward propagation (cf. Fig. 8) of this evening sector FLR is also not compatible with a flank waveguide mode, where propagation should be antisunward.

Figure 7 shows the high pass filtered $x$-component magnetometer data for this event. Low frequency oscillations, with a period of about $13 \mathrm{~min}$, are evident at all stations. Power spectra were computed using these data and a time-domain window $2 \mathrm{~h}$ long, centered at 04:26 UT. The data were detrended (linear trend) and high pass filtered $(0.5 \mathrm{mHz})$ before computing the power spectrum from a discrete Fourier transform. No spectral smoothing was used, and so the effective resolution of the spectrum is $\Delta f=1 /(2 \mathrm{hr})=$ $0.14 \mathrm{mHz}$. The spectrum shows a distinct peak at approx- 

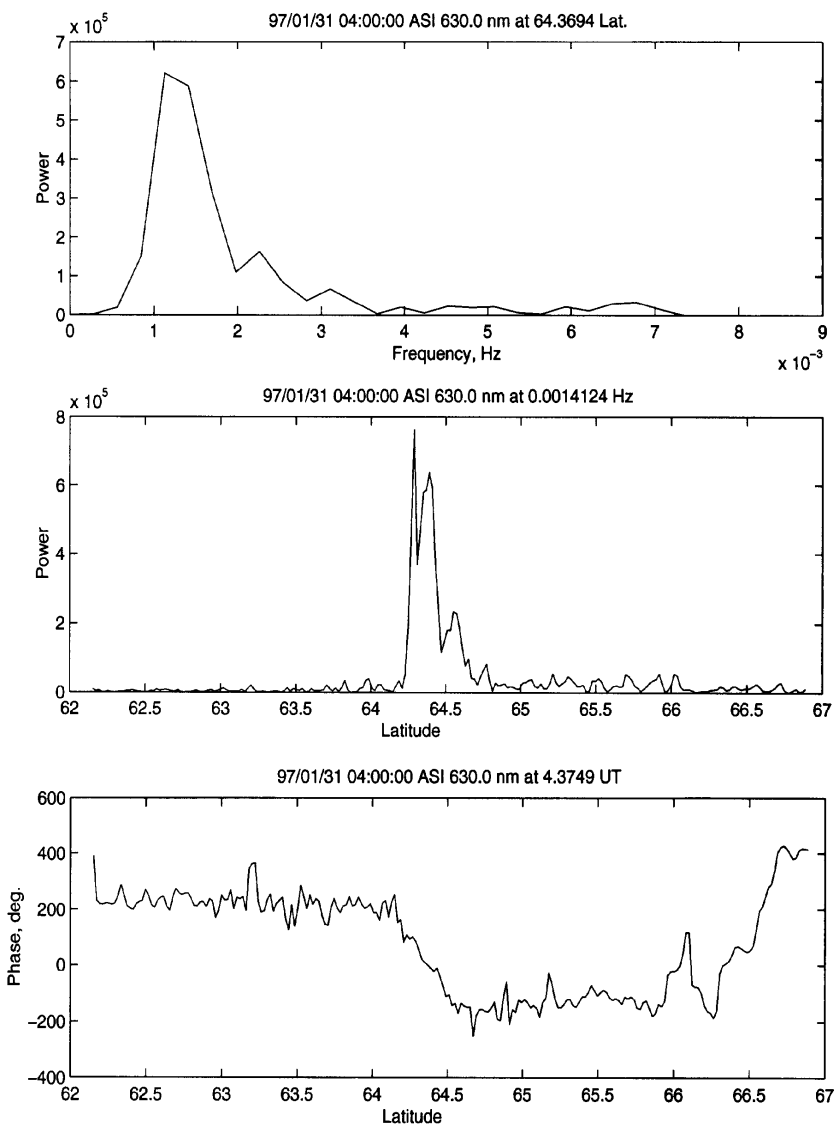

Fig. 9. Power and phase as a function of latitude for the $1.3 \mathrm{mHz}$ spectra of ASI, $630.0 \mathrm{~nm}$ luminosities recorded at a longitude of 337 degrees.

imately $1.3 \mathrm{mHz}$, corresponding to the 13 min periodicity seen in the MPA data. The true width of the spectral peak of the FLR is not resolved with this $2 \mathrm{hr}$ window, and longer time series indicate a spectral width substantially less than $0.14 \mathrm{mHz}$.

Figure 8 shows the $630.0 \mathrm{~nm}$ ASI images associated with the FLR. By inspecting the temporal sequence, the westward propagation becomes apparent. In comparing the images with the schematic in Fig. 2, the top image in Fig. 8, at a longitude of 334 degrees, corresponds to Phase 2. In the third image from the top, a longitude of 338 degrees corresponds to Phase 4. Arc-1 marks the poleward region of upward FAC (Fig. 2, Phase 4), and arc-2 marks the equatorward region of upward FAC. The maxima in the emissions for these two arcs are separated by approximately $30 \mathrm{~km}$ in latitude, and taking into account the slight tilt of the FLR center (changing latitude with longitude), the separation is near $25-30 \mathrm{~km}$. The width of the individual arcs was $10 \mathrm{~km}$, allowing us to estimate a full FLR width of approximately $35-40 \mathrm{~km}$. Comparison of the optical images indicates a westward phase velocity of approximately $0.024 \pm 0.002$ degrees/s. The azimuthal phase velocity was estimated by plotting the maximum (in latitude) intensity of the arc as a function of longitude and then determining the longitude of the maximum slope. The azimuthal velocity was estimated from the longitudinal motion of this maximum in the sequence of three ASI images at the top of Fig. 8. Noting the resolution of the spectra, this corresponds to an azimuthal $m$-value of 17-22 (assuming an azimuthal or $\phi$ dependence of the form $e^{i m \phi}$ ). The westward propagation of the arc structures is quite clear in Fig. 8, and a quick visual inspection can confirm an approximation to the azimuthal velocities. These relatively large $m$-values potentially raise a problem with the model of a compressional driver for the FLR. Coupling of the compressional and shear Alfvén, FLR mode is most efficient for low $m$-values, typically less than 10 (Samson et al., 1992a). However, as pointed out by Kivelson and Southwood (1986), the coupling efficiency depends on the steepness of the Alfvén speed gradient. Sharp gradients will give stronger coupling at higher values of $m$. In particular, it has been suggested recently by Lee et al. (2001), that FLRs may be excited on strong gradients near the inner edge of the plasma sheet. This location is consistent with the FAST event described in this study.

Aside from the problem of coupling to the compressional driver, our analysis shows that the optical emissions associated with the FAST event have all the characteristic features of a shear Alfvén FLR. Figure 8 indicates an extended longitudinal length of the arcs of about $18^{\circ}$. Correlating luminosity with $j_{\|}$, we note that ionospheric (and magnetospheric) flows should be predominantly azimuthal, and the electric field is in the transverse (latitudinal) $x$-direction. Noting the estimated $m$-value and the features above, it is unlikely that this FLR is connected with a drift Alfvén wave, since in this mode the electric field is azimuthal and the fluid velocities are in the transverse direction (Chan et al., 1994). Consequently, it is very unlikely that a drift Alfvén mode can be used to explain the very low frequencies of the FLR, and we shall later consider a model with stretched field lines.

To characterize the latitudinal structure of the FLR, the time series of the $630.0 \mathrm{~nm}$ emissions at 337 degrees longitude were Fourier transformed at each latitude, to measure the power and phase as a function of frequency. Detrending and filtering were the same as that done with the magnetometer data. The optical features of the FLR appeared only over a limited time period, from about 04:00 to 04:45 UT, and consequently, we have computed the spectral data over the interval from 04:00 to 05:00 UT. The power spectrum at the latitude of the center of the FLR is shown in the top panel of Fig. 9. A maximum near $1.3 \mathrm{mHz}$ is evident, and is compatible with the magnetometer spectra. Note, however, that the spectral resolution is less accurate, only $0.28 \mathrm{mHz}$. The middle panel shows the power at $1.4 \mathrm{mHz}$ as a function of latitude, and clearly indicates the latitudinal localization of the FLR. Depending on where the cutoff in the spectral power is selected, the width of the FLR is on the order of $50 \mathrm{~km}$ or less. This width is compatible with the FAST observations.

We would like to point out a number of further interesting features in these data. First, the latitudinal profile of the power is asymmetric, with a tail on the poleward side. Second, the phase shift through the resonance is substantially greater than $180^{\circ}$, even though the data (optical and magne- 
tometer) indicate the existence of only one FLR at $1.3 \mathrm{mHz}$. As we show later, these observations might indicate dispersive effects, which cause the propagation of the shear mode across magnetic shells.

\section{FLR model}

In this section, we present a model of a driven and nonlinearly saturated standing shear Alfvén wave, and will demonstrate that it readily explains many of the observed features of the $1.3 \mathrm{mHz}$ FLR observed by CANOPUS and FAST. The model incorporates the following important elements of shear Alfvén wave physics:

- It describes the eigenmode structure and the eigenfrequency of a standing shear Alfvén wave on geomagnetic field lines. We consider the wave fields as a small perturbation to the ambient magnetic field, which is described using the empirical T96 model (Tsyganenko, 1996).

- The shear wave is driven by a monochromatic compressional wave of an appropriate frequency, which is considered as an external, prescribed driver. The amplitude of this driver is the only free parameter in the model. It is adjusted in such a way that the wave saturated amplitude near the ionosphere agrees with observations.

- The saturation of the driven shear wave is due to three effects: (i) ionospheric damping described by the height-integrated Pederson conductivity; (ii) thermal effects which provide wave dispersion through finite ion gyroradius effects and electron parallel kinetics (see, for example, Goertz, 1984); (iii) shear wave ponderomotive forces which detune the wave frequency from the driver by redistributing the plasma density along the field line.

- The parallel electric field is generated self-consistently within the model due to the nonlocal electron thermal response, assuming that the electron bounce frequency in the geomagnetic field is larger than the wave frequency. The parallel electric current is generated by the dominant (nondispersive) part of the shear wave. Then the parallel electric field which is needed to support the parallel current is calculated. Though this parallel electric field is a second order effect in our model, which does not change the wave eigenstructure; it makes a significant contribution to wave dispersion and in this way defines the saturated wave amplitude. One consequence of the parallel electric field is that it will accelerate electrons and ions into the ionosphere, producing optical emission, and initiating a feedback effect on the wave amplitude by changing the Pedersen conductivity.

In the following, we briefly describe all the individual elements of the model and compare the results it produces with ground and satellite observations.

\subsection{FLR eigenstructure and eigenfrequency}

The FLR model used in this study considers field and current structures in the vicinity of a given geomagnetic shell as small perturbations. Therefore, the profile of the magnetic shell (assuming azimuthal symmetry of the geomagnetic field), and the distribution of the magnetic field and plasma density, are the input characteristics of the model. This input is used in the calculation of the eigenfrequency and eigenmode structure of the toroidal shear Alfvén wave along the magnetic field line, although the wave amplitude and its radial profile (in the direction perpendicular to the magnetic surface) will be calculated later using a higher order approximation. The procedure for calculating the eigenmodes in a curvilinear geometry has been explained in detail in previous publications (Chan et al., 1994; Rankin et al., 2000). In this section, we discuss the approximate FLR mode structure for the 31 January 1997 event.

One of the points that Lotko et al. (1998) raised, is the problem of the very low frequency of the observed FLR. The latitude, 65.9 degrees, of the observations corresponds to a magnetic field line mapping to $5.9 R_{E}$ in the equatorial plane of a dipolar magnetic field, where the magnetic field is $90 \mathrm{nT}$. Correspondingly, the calculations of Lotko et al. (1998) give a frequency for the FLR near $11 \mathrm{mHz}$, assuming the plasma density in the equatorial plane is about 1 particle per c.c. Nevertheless, numerous observations (Samson et al., 1992a; Waters et al., 1996) indicate that the FLR frequency at this latitude is much lower, at times below $2 \mathrm{mHz}$. Chan et al. (1994) have clearly shown that a stretched field topology in the magnetotail, associated with near-Earth $\left(8-12 R_{E}\right.$ tailward) pressure gradients, can lead to FLR eigenmodes with much lower frequencies. Rankin et al. (2000) used the magnetic field line profiles based on the T96 (Tsyganenko, 1996) magnetic field model to illustrate this point. Their calculated FLR frequency for solar wind conditions that was used to model the 31 January 1997 FAST event (pressure $3 \mathrm{nPa}$, $D_{s t}=-30 \mathrm{nT}, B_{y}=0$, and $B_{z}=-3 \mathrm{nT}$ ) is $1.3 \mathrm{mHz}$ for the midnight sector, at a magnetic latitude of 65.9 degrees. The damping time is about $60 \mathrm{~min}$ for a Pedersen conductivity $\Sigma_{P}=5 \mathrm{~S}$. According to the T96 model, this magnetic field line maps to a distance of $16.1 R_{E}$ in the equatorial plane, where the magnetic field magnitude is $4.3 \mathrm{nT}$. In contrast, the field line resonances observed $4 \mathrm{hrs}$ prior to the FAST pass, were at higher latitudes, 72.5 and 73.5 degrees. At this time the field lines are closer to the dayside, and should show less stretching. Frequencies calculated with a dipole model are 2 and $2.5 \mathrm{mHz}$, respectively, agreeing with measured values. This suggests that significant field line stretching had occurred during this time period, as the observations move from local evening to local midnight. The CANOPUS magnetometers show considerable substorm activity in the magnetotail prior to local midnight, indicating energy storage and stretching of field lines.

The observations from CANOPUS demonstrate that the observed $1.3 \mathrm{mHz}$ FLRs are consistent with a stretched field topology. This is more clearly seen in the optical data. In 

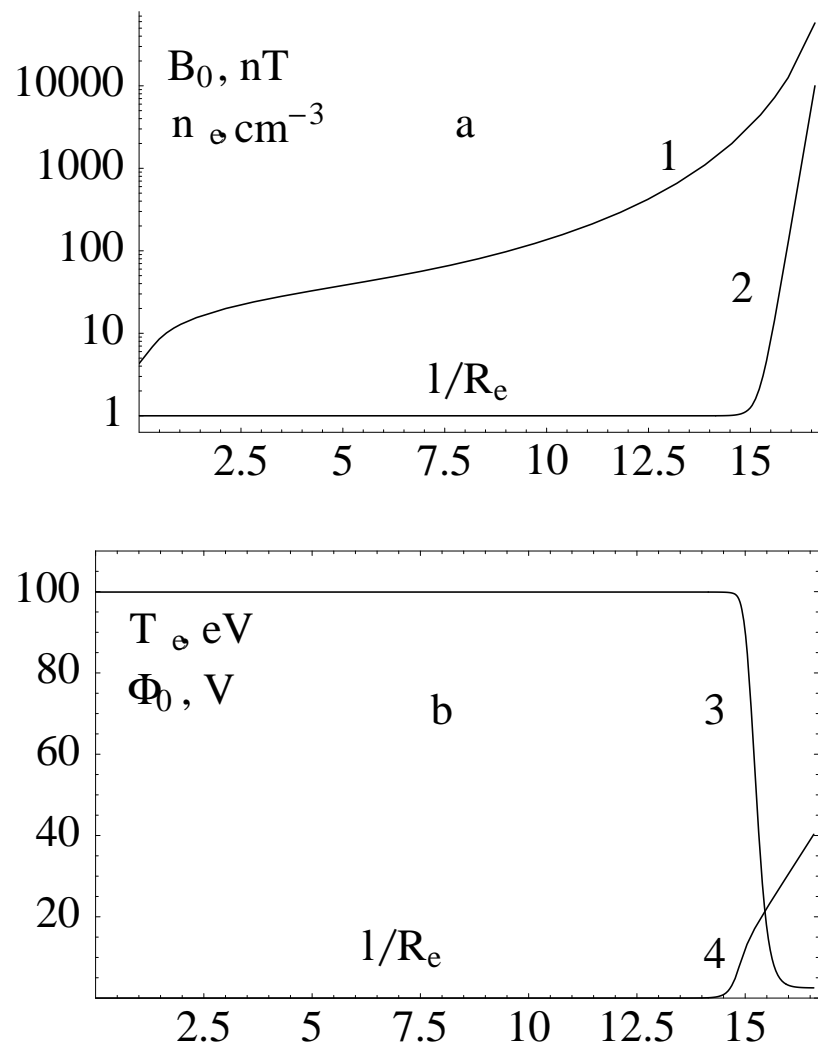

Fig. 10. Variation of the ambient magnetic field (1), plasma density (2), electron temperature (3), and the electrostatic potential (4), along the magnetic field line. The equatorial plane is at $l=0$, and the ionospheric end corresponds to $l_{\max }=16.6 R_{E}$, the FAST altitude corresponds to $l=16 R_{E}$.

Fig. 6, we can see that the FLRs are on the poleward border of a band of $486.1 \mathrm{~nm}$ emissions. These emissions are due to energetic $(10 \mathrm{~s}$ of $\mathrm{keV})$ protons that are scattered into the loss cone due to nonadiabatic trajectories in the equatorial plane of the near-Earth plasma sheet. Consequently, the radius of curvature of the field lines in the equatorial plane must be comparable to the proton gyroradius in that region. Indeed, it is shown by Rankin et al. (2000) that the curvature of the resonance line in the equatorial plane, $R_{c}=0.4 R_{E}$, is smaller than the gyroradius of $1 \mathrm{keV}$ protons, $\rho_{i}=0.5 R_{E}$.

\subsection{Time evolution of the driven FLR}

Now we address the problem of the spatial and temporal evolution of the driven FLR. As explained above, the FLR eigenfrequency, $\omega$, and the eigenfunction, $S(l)$, along the magnetic field line, are found for a cold plasma. Here, we consider higher order effects (dissipation, thermal motion, and nonlinearity) which are important for the FLR formation and its subsequent temporal evolution and spatial structuring. In order to consider these higher order effects iteratively, we represent the wave magnetic field in the envelope approximation, $B_{y}=b_{y}(x, t) S(l) \sin \left(\omega_{0} t-m \phi\right)(m$ is the azimuthal mode number, and $\omega_{o}$ is the driver frequency) and assume that the wave amplitude $b_{y}$ changes slowly during one wave period. The equation describing the evolution of the wave amplitude in time, and in the transverse direction $x$, has been derived by Frycz et al. (1998) and Rankin et al. (1999a),

$\partial_{t} b_{y}-i \frac{\omega_{0}}{2} \delta \partial_{x}^{2} b_{y}=i(\delta \Omega-\Delta \omega) b_{y}+\frac{\omega_{0}}{2} R$,

where each coefficient is evaluated by using the zero-order eigenfrequency and eigenfunction, and the profiles of the plasma density and temperature along the magnetic field line. The second term on the left-hand side of the previous equation accounts for dispersive effects. The coefficient $\delta$ is the sum of the ion and electron contributions. The ion contribution is proportional to the square of the ion gyroradius averaged over the magnetic field line length, $\left\langle\rho_{i}^{2}\right\rangle$. The electron contribution is proportional to the square of the electron inertia length, $\left\langle\lambda_{e}^{2}\right\rangle$, if the characteristic electron bounce frequency, $\omega_{b}=V_{t e} / l_{\max }$ is smaller than the wave frequency, or it is of the order of $\left\langle\left(\lambda_{e} \omega_{b} / \omega_{0}\right)^{2}\right\rangle$, if $\omega_{b}>\omega_{0}$. The latter effect dominates, since for the present case the electron temperature is $100 \mathrm{eV}$, the electron thermal velocity $V_{t e}=4200 \mathrm{~km} / \mathrm{s}$, the field line length $l_{\max }=33 R_{E}$, and the electron bounce period, $1.7 \mathrm{~min}$, is much shorter than the FLR period of $12.8 \mathrm{~min}$. In that case the electrons respond nonlocally and one has to use kinetic theory to evaluate the wave dispersion. The electron kinetic effects are described in the next section. Here, we mention that $\delta \approx 0.018 R_{E}^{2}$ for the present FLR parameters. The linear frequency mismatch, $\Delta \omega$, on the right-hand side of Eq. (1) is a complex quantity. Its real part describes linear frequency detuning due to the radial dependence of the FLR eigenfrequency, $\omega(x)$, on the chosen magnetic shell. Its imaginary part arises due to wave damping. By choosing the perpendicular coordinate $x=0$ at the resonance magnetic shell, $\omega(0)=\omega_{0}$, one has $\Delta \omega=x \omega_{0} / 2 l_{\omega}-i \gamma$, where $l_{\omega}=3.2 R_{E}$ is the gradient scale length of the Alfvén wave frequency, $\gamma=0.02 \omega_{0}$, with values calculated for the conditions described in Rankin et al. (2000). The damping of the FLR is due to the Pederson conductivity at the ionospheric ends of the magnetic field line.

The nonlinear frequency shift, $\delta \Omega$, in Eq. (1) is due to density depletions that are created by the ponderomotive force of the standing shear Alfvén wave. It is roughly proportional to $\left|b_{y}^{2}\right|$. The amplitude of the driver, $R=0.3 \mathrm{nT}$, has been chosen such that the saturated wave amplitude, $b_{\text {sat }} \approx 60 \mathrm{nT}$, at an altitude of $4000 \mathrm{~km}$ agrees with the FAST observations. This is the only parameter that must be adjusted. All other fields and currents have been calculated self-consistently from the derived $b_{y}$. In particular, the parallel electric current is proportional to the transverse derivative, $J_{\|} \sim \partial_{x} b_{y} S(l) \sin \left(\omega_{0} t\right)$, and the transverse electric field $E_{x}$ is proportional to the derivative of $B_{y}$ along the field line. It is important to realize that $E_{x}$ has two components, the inphase component, $E_{x}^{p} \sim\left(V_{A}^{2} / \omega\right) b_{y} \partial_{l} S(l) \cos \left(\omega_{0} t-m \phi\right)$ and the quadrature component $E_{x}^{q} \sim\left(b_{y} / \mu_{0} \Sigma_{P}\right) \sin \left(\omega_{0} t-m \phi\right)$. The latter arises due to finite Pedersen conductivity (Samson et al., 1996) and dominates at low altitudes, such as iono- 
spheric and FAST altitudes. For a perfectly conducting ionosphere, there is no quadrature field that accounts for wave damping and provides a net Poynting flux from the FLR into the ionosphere. It is important to remind the reader that the dispersive, dissipative, and nonlinear effects make additive contributions to the envelope Eq. (1). The relation between these effects is unspecified, because each of them is evaluated using the zeroth-order FLR characteristics. A fully selfconsistent, nonlinear kinetic model of FLRs has not yet been developed.

The backgound profiles of the magnetic field, plasma density and the electron temperature are shown in Fig. 10. The low minimum of the magnetic field amplitude near the equator is due to field line stretching. The rapid increase in the plasma density, and decrease in the electron temperature near the ionosphere, are due to oxygen expanding from the ionosphere in the presence of the Earth's gravitational field. The field profiles along the magnetic field line are found from the solution to the eigenmode equation for the SAW and are shown in Fig. 11. The increase in the azimuthal magnetic field and the parallel electric current density is due to convergence of the magnetic field lines near the ionosphere. In fact, the current is almost divergenceless at the altitudes represented in Fig. 11. In the transverse electric field, the quadrature component dominates at low altitudes. At FAST altitudes, and for typical ionospheric conductivities, the quadrature electric field from the model is almost five times larger than the in-phase field. This is an important point for interpretating the electric field data from FAST. Though we have adjusted only the amplitude of the azimuthal magnetic field, the amplitude of the parallel current is also in agreement with the observations. The calculated radial electric field shown in Fig. $11 \mathrm{c}$ is, however, about $10 \mathrm{mV} / \mathrm{m}$, and is approximately 10 times smaller than measured. We do not have a complete explanation for this; however, one should notice that the high-amplitude (up to $150 \mathrm{mV} / \mathrm{m}$ ) radial electric field measured by FAST instruments corresponds to small spatial scales of the order of $1 \mathrm{~km}$ or less. The large-scale component (also seen in the FAST magnetic field data), which can be estimated after filtering out the high-frequency component, is of the order of $10 \mathrm{mV} / \mathrm{m}$, and is in agreement with the model.

Figure 12 shows the radial profiles of the azimuthal magnetic field taken near the ionospheric end of the magnetic field line. The scales should be increased two times in mapping these plots to FAST altitudes of $4000 \mathrm{~km}$. The time corresponds to $t=50 \mathrm{~min}$ in Fig. 13, well after the saturation of the resonance. The magnetic field amplitude has a characteristic maximum at the resonance position, $x=0$, which is broadened northward due to wave dispersion. The phase exhibits a $180^{\circ}$ shift across the resonance, and is very similar to the Fourier spectra from the optical data (Figs. 5, 7, and 9). An additional phase shift north from the resonance $(x \approx 30 \mathrm{~km})$ is an indication of wave propagation due to thermal electron dispersion.

The characteristic FLR radial scale for the present conditions is determined by the balance between the linear fre-
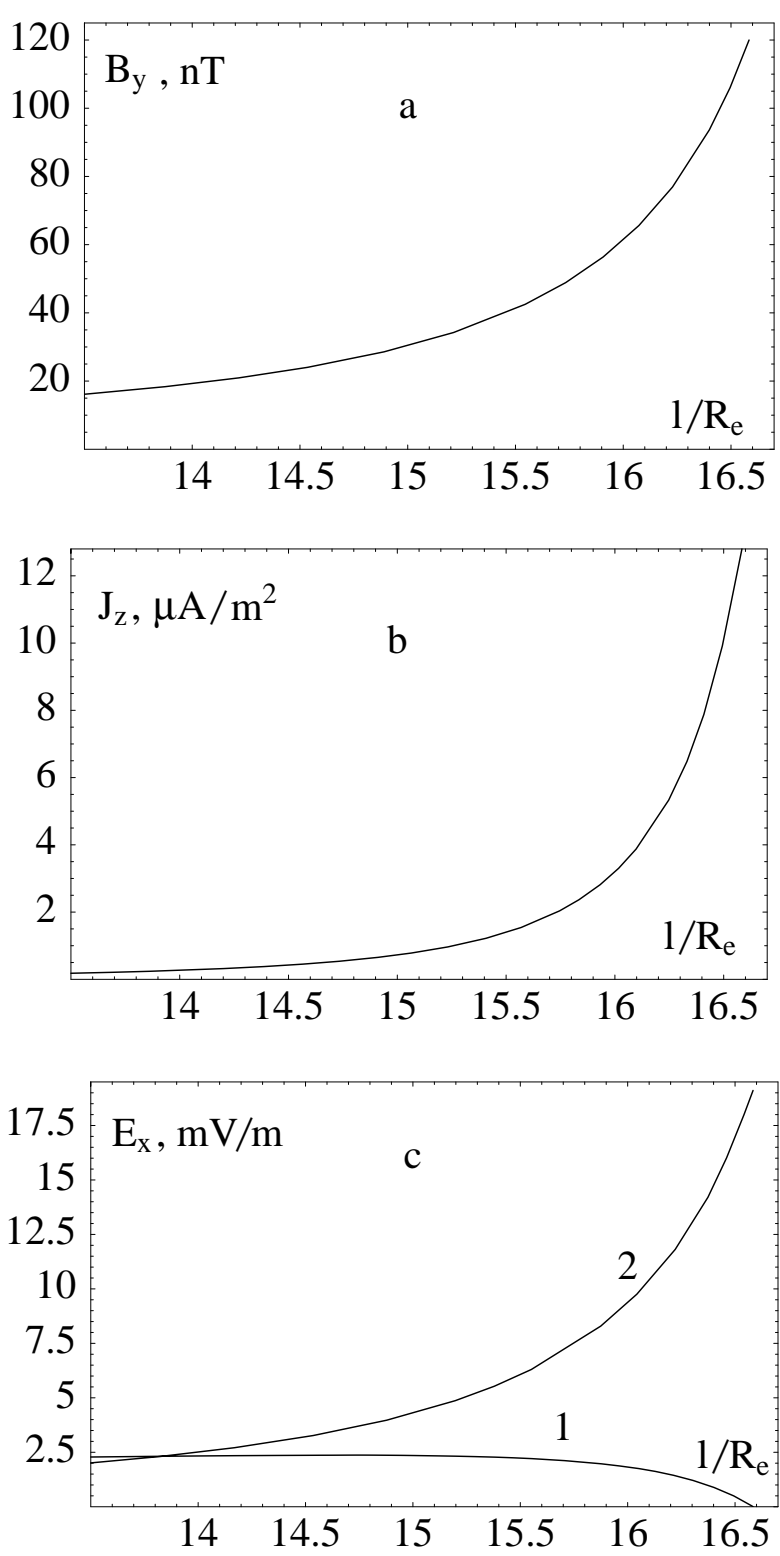

Fig. 11. Dependence of the azimuthal magnetic field (a), parallel electric current (b), and transverse electric field ((c), in-phase, 1, and quadrature, 2, components) on the coordinate along the magnetic field line. Only part of magnetic field line close to the ionosphere is shown. Amplitudes are taken at the time of FLR saturation. The ionospheric end corresponds to $l_{\max }=16.6 R_{E}$, the FAST altitude corresponds to $l=16 R_{E}$.

quency detuning scale length, $l_{\omega}=3.2 R_{E}$, and dispersion, $\delta \approx 0.018 R_{E}^{2}$. At the equatorial plane, $\Delta x_{e q}=\left(l_{\omega} \delta\right)^{1 / 3}=$ $0.4 R_{E}$ (Frycz et al., 1998), and one needs to divide this result by the radial compression factor, which is approximately 340 for the chosen magnetic field line. Then one arrives at the estimate $\Delta x \sim 20 \mathrm{~km}$ at the altitude of FAST and $\Delta x \sim 10 \mathrm{~km}$ above the ionosphere. The corresponding current amplitude, $J_{\|} \sim B_{y} / \mu_{0} \Delta x$, is of the order of $10 \mu \mathrm{A} / \mathrm{m}^{2}$ above the ionosphere. These numbers are in agreement with the model cal- 

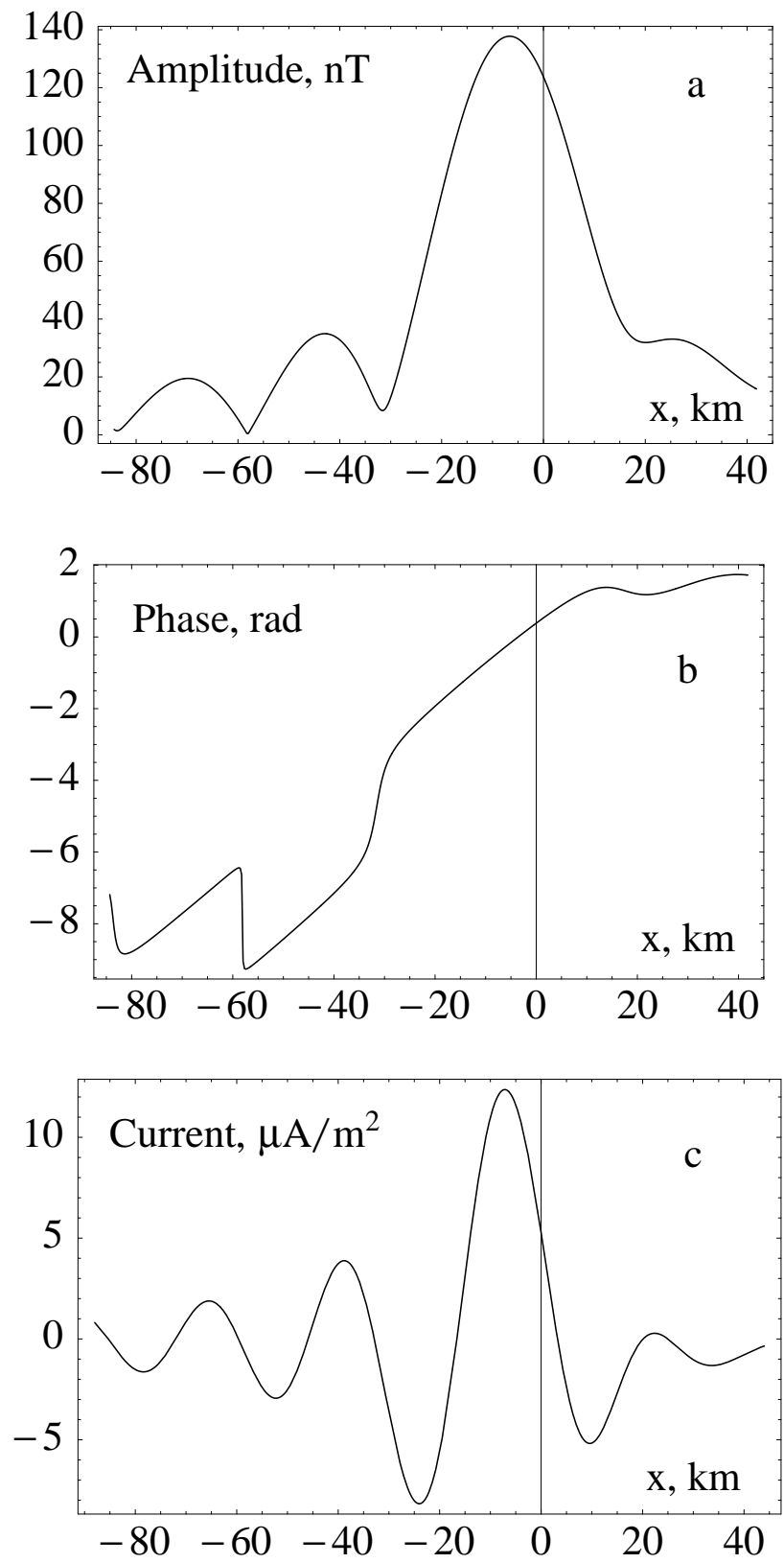

Fig. 12. Snapshort of the transverse profiles of the magnetic field amplitude (a), phase (b), and the parallel electric current from the dispersive FLR model at the end of magnetic field line. The FLR position corresponds to $x=0$, poleward is to the left.

culations in Figs. 11 and 12 and with the observations shown in Figs. 6-9. The parallel current in Fig. 12c has a structure that corresponds roughly to Phase 2 in Fig. 2, although there are additional smaller peaks on the poleward side due to dispersive effects.

The temporal evolution of the resonance is presented in Fig. 13. We have assumed that a driver of constant amplitude has been switched on at time $t=0$. The time of the FLR saturation due to dispersive effects, $\omega t_{\mathrm{sat}} \sim 2\left(l^{2} / \delta\right)^{1 / 3} \approx 16$, is less than three wave periods, as seen in Fig. 13. After this time the wave amplitude at the resonance stays approx-

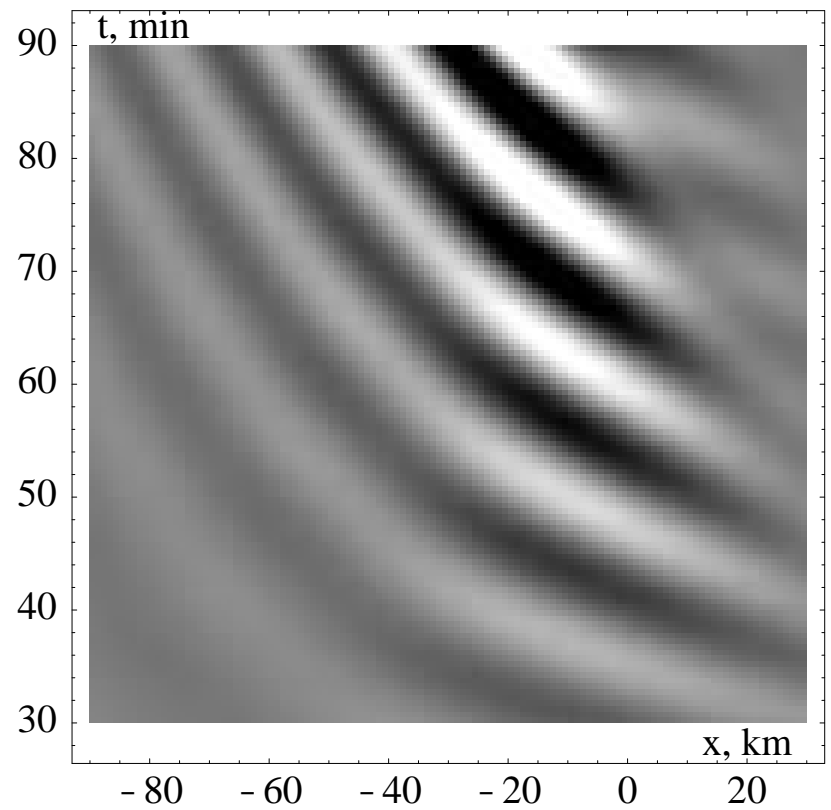

Fig. 13. Temporal and $x$-dependence of the amplitude of the parallel electric current above the ionosphere. The FLR position corresponds to $x=0$, poleward is to the left.

imately constant. The resonance periodically emits weaker current sheets, which propagate slowly northward and carry energy out of the resonance. The transverse velocity is determined by dispersion and is of the order of a few $\mathrm{km} / \mathrm{min}$ in this example.

In the model for this event, the FLR evolution is marginally linear. The nonlinear contribution to the phase shift is a few times less than the dispersive term. Simulations with a two-three times stronger driver (which seems a realistic case, because the present event corresponds to a relatively quiet period of magnetospheric activity), demonstrate more violent wave evolution, with formation of narrow current sheets moving slowly in the northward direction (Rankin et al., 1999a). These current sheets (solitons) are accompanied by plasma density depletions created by the ponderomotive force. The cavities are extended along field lines to a few $R_{E}$ from the ionosphere and have latitudinal widths as small as a few km (Rankin et al., 1999b).

\subsection{Electron kinetics and parallel electric field}

The FLR dispersion discussed above is due to the parallel electric field generated by electron thermal motion along the magnetic field line. Under the present conditions, where the electron bounce frequency is larger than the FLR frequency, the electron response is nonlocal and has to be calculated using kinetic theory, as we will explain below. We find that local dispersive effects, including electron inertia and ion gyroradius, cannot give the required parallel potential drop for the FAST conditions (Rankin et al., 1999a, 1999c).

Lotko et al. (1998) considered both dispersion due to electron inertia and anomalous resistivity in constructing a FLR 


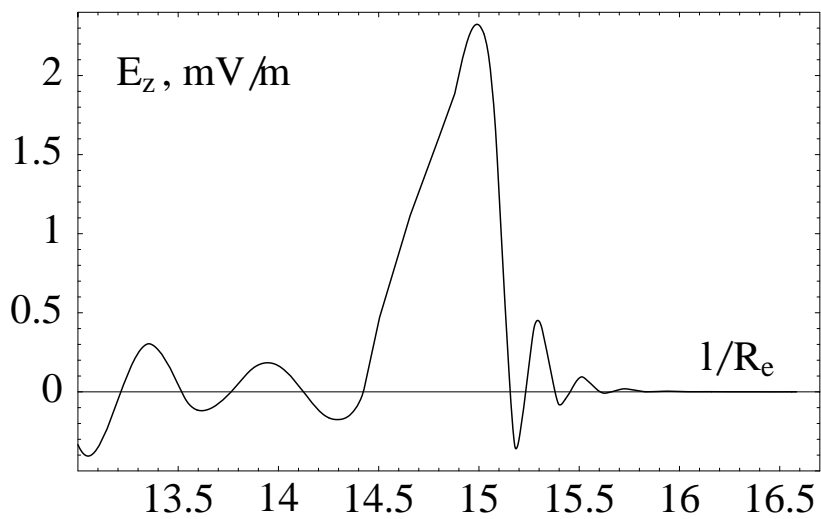

Fig. 14. Wave parallel electric field distribution along the field line near the ionosphere. The ionospheric end corresponds to $l_{\max }=$ $16.6 R_{E}$, the FAST altitude corresponds to $l=16 R_{E}$. The parallel electric field is located $1 R_{E}$ above the FAST orbit.

model. Although their model gives a very good match to the overall form of the field-aligned current and the finer scales in the perpendicular fields, its physical background is contradictory. First, as has been explained above, electron inertia does not contribute to the FLR dispersion, because the electron thermal velocity is larger than the Alfvén velocity everywhere on the field line, except in short sections near the ionosphere. Second, the anomalous resistivity invoked in their model, $v_{e f} \sim 10 s^{-1}$, on the length about $1000 \mathrm{~km}$, corresponds to a FLR damping time of the order of a few minutes. This is a prohibitively short time for a realistic FLR with a period of $12.8 \mathrm{~min}$ (although it is longer than the 1.3min period of the dipolar FLR used in Lotko's simulations).

An alternative resolution to the problem of small fieldaligned potential drops lies in the consideration of the electron kinetic effects, including mirroring and precipitation. Static models considering particle mirroring effects have already been constructed by Knight (1973), Fridman and Lemaire (1980), and Chiu and Schulz (1978), amongst others. In particular, Chiu and Schulz (1978) have treated kinetic electrons and ions that are coupled via Poisson's equation. However, such a model is not applicable to FLRs where ions and electrons play completely different roles. Ions move slowly along field lines during one FLR period. Consequently, they respond locally by feeding the field-aligned current through the polarization drift. Conversely, electrons carry the parallel current bounce many times during the wave period between turning points along the field line.

We consider a time dependent kinetic electron response to a given oscillating field-aligned current. The dependence of the current along the field line is found from the solution to the cold and collisionless (ideal MHD) eigenmode equation, as described above. This FAC is shown in Fig. 11b, along with the azimuthal magnetic and transverse electric fields. Once the FACs have been prescribed, the field-aligned electric fields that are needed to supply such a current, are computed from the electron kinetic equation by accounting for their orbits in the geomagnetic field, $B_{0}(l)$, and the quasi- static potential, $\Phi_{0}(l)$, that arises due to ambient density inhomogeneities.

The procedure for determining parallel electric fields has the following two steps. First, we solve the one-dimensional electron gyrokinetic equation (Antonsen and Lane, 1980) for the electron perturbation driven by a periodic electric field. The perturbation of the electron distribution function along the field line, $\delta f_{e \pm}$, averaged over the electron gyroperiod and oscillating at the frequency $\omega$, is given by

$\left(-i \omega+ \pm v_{\|} \partial_{l}\right) \delta f_{e \pm}= \pm e E_{\|} v_{\|} \partial_{w} f_{e 0}$,

where $f_{e 0}(w)$ is the background electron distribution, which depends on the total electron energy, $w=m_{e} v^{2} / 2-e \Phi_{0}$, and signs \pm give the direction of the electron velocity along the geomagnetic field. Next, we introduce the antisymmetric part of the distribution function $\delta f_{e a}=\left(\delta f_{e+}-\delta f_{e-}\right) / 2$, calculate the electric current, $J_{\|}=-e \int d^{3} v v_{\|} \delta f_{e a}$, and represent the current-field relation in the form of a nonlocal Ohm's law, $J_{\|}(l)=\int d l^{\prime} \sigma\left(l, l^{\prime}\right) E_{\|}\left(l^{\prime}\right)$, where $\sigma$ is a nonlocal conductivity that relates the magnitude of the electric potential at the point $l^{\prime}$ to the parallel electric current at the point $l$. Then, given $J_{\|}$, the inverse problem is solved to find $E_{\|}$. The details and calculations are rather tedious, because the contribution of various open and closed electron orbits need to be taken into account (Rankin et al., 1999c; Tikhonchuk and Rankin, 2000).

The boundary conditions for the electrons at the ends of the magnetic field line allow the current to penetrate to the ionosphere, but conserve the number of electrons. In particular, the current continuity condition at the ionosphere, $\partial J_{\|} / \partial l=0$, allows the parallel current to feed ionospheric Pedersen currents. This is an important condition that causes the wave reflection that is required to set up the FLR. On the other hand, the condition of charge conservation at the ionosphere, $\partial \rho_{c} / \partial t=0$, is a convenient assumption that does not affect the FLR dynamics significantly.

Since the current is defined by the antisymmetric part of the electron distribution function, we require $\partial_{l} \delta f_{e a}=0$ at the ionospheric ends of the magnetic field line. The charge density is defined by the symmetric part of the electron distribution, $\rho_{c}=-e \int d^{3} v \delta f_{e s}$, where $\delta f_{e s}=\left(\delta f_{e+}+\delta f_{e-}\right) / 2$, and we require at the ionospheric ends that $\delta f_{\text {es }}=0$. Therefore, there is no problem with the flux tube plasma depletion that was found to be crucial in static kinetic models. Our open boundary conditions may result in a sheath potential at the ionosphere. However, it can be shown to be much smaller than the wave potential, as it is inversely proportional to the density.

Figure 14 shows the distribution of the parallel electric field along the magnetic field line, for the plasma conditions and parallel current distribution presented in Figs. 10 and $11 \mathrm{~b}$, respectively, and at the radial position of the maximum current of the saturated FLR. The maximum of about $1 \mathrm{mV} / \mathrm{m}$ is located at an altitude of $1.6 R_{E}$ above the ionosphere, where the plasma density starts to increase (earthward), due to the oxygen contribution from the ionosphere. For our chosen ambient profiles, this maximum is above the 
altitude of FAST. Nevertheless, the maximum is very sensitive to density gradients, which are not completely accounted for in our model. The total potential drop, $\Phi_{\|}=-\int E_{\|} d l$, is a more robust parameter that is not too sensitive to local conditions. Its calculated amplitude is of the order of $6 \mathrm{kV}$. This potential is more than adequate to accelerate electrons to the energies measured by the FAST electron detectors, and is large enough to produce the discrete arcs seen in ASI images (Fig. 8).

A more detailed analysis of the electric field generation in Rankin et al. (1999c) and Tikhonchuk and Rankin (2000), shows that the width of the peak of the parallel electric field, $\Delta l$, is of the order of the characteristic density scale length near the ionosphere, $h=C_{S}^{2} / g$, where $C_{S}$ is the ion acoustic velocity above the ionosphere and $g$ is the gravitational constant. Therefore, the electric field is sensitive to the electron temperature above the ionosphere. The full potential drop can be estimated by a current-voltage relation:

$J_{\|}=C\left(e^{2} n_{e} \omega l_{\max } / T_{e}\right) \Phi_{\|}$,

where $n_{e}$ and $T_{e}$ are the electron density and temperature at the field location and $l_{\max }$ is the length of the magnetic field line. The coefficient $C \sim \ln \left(n_{\text {ion }} / n_{\text {eq }}\right)$ accounts for the electrostatic potential along the field line due to the density increase from the equatorial level, $n_{e q}$, to the ionospheric level, $n_{\text {ion }}$ (cf. Fig. 10a). The coefficient $C$ is smaller if the electrostatic potential is calculated, taking into account the contribution of cold electrons from the ionosphere. This is not considered here, although it is clear from Eq. (3) that the effect of cold electrons will be to reduce the parallel potential drop. The current-voltage relation (Eq. 3 ) is proportional to the wave frequency (or, in other words, the current is proportional to the time derivative of the potential) and, therefore, it is completely different from the static current-voltage relation derived by Knight (1975), $J_{\|}=\left(e^{2} n_{e} / m_{e} V_{t e}\right) \Phi_{\|}$. The parallel potential of Knight is in phase with the parallel current, while the potential given by Eq. (3) is 90 degrees out of phase with the current. Consequently, there is no additional dissipation in our model of FLRs. However, what remains to be done is to calculate the flux of fast electrons that will be accelerated by the field-aligned potential drop predicted by our model. This will lead to real dissipation, although the calculation is non-trivial, and is left for future studies. Also, Eq. (3) predicts that the potential is larger than the Knight relation by the ratio of the electron bounce frequency to the wave frequency.

Generation of parallel electric fields due to electron thermal motion has important consequences for wave dynamics. First, it affects wave dispersion that is responsible for wave propagation across magnetic surfaces. The dispersion coefficient calculated from the parallel electric field distribution shown in Fig. 14, $\delta \approx 0.018 R_{E}^{2}$, has been substituted into the envelope wave Eq. (1), where it defines the level of FLR saturation and the radial structure of the fields. Second, the parallel electric field creates a force which expels electrons from large field locations. Due to quasi-neutrality (which holds to a very good accuracy, because the width $\Delta l$ of the potential is much larger than the Debye length), ions will also be displaced along the field line, and, therefore, the generation of parallel electric fields also produces density perturbations. Assuming that ion inertia is not important, that is, the characteristic time of ion local response, $\Delta l / V_{t i}$, which is less than $1 \mathrm{~min}$, is smaller than the wave period, the amplitude of the density depletion can be estimated from the ion pressure balance along the field line, $\delta \rho / \rho_{0} \sim e \Phi_{\|} / T_{i}$. This estimate results in very large density perturbations, $\delta \rho / \rho_{0} \sim 1$ for the present parameters. One should be aware that the periodic density perturbation shown due to parallel electric fields is different and complementary to the quasi-static density depression produced by the wave ponderomotive force. However, both types of density perturbations may be excited and their relative importance depends on the wave nonlinearity. In the marginally linear regime, which corresponds to the conditions of the event of 31 January 1997, the linear density perturbation is larger than the ponderomotively-driven perturbation. However, for a driver amplitude only a few times larger, the ponderomotive effects will dominate.

\section{Conclusions}

The comparison between CANOPUS - FAST observations and the theoretical model discussed above demonstrates that FLRs can produce auroral arcs. In particular, the CANOPUS data show that the associated FAST event (Lotko et al., 1998) was an observation of an electron accelerator region coincident with a $1.3 \mathrm{mHz}$ FLR. The FLR was seen in the magnetometer, MPA, and ASI data. The FLR had all the standard features of a toroidal field line resonance, though the estimated $m$-value (17-22) is somewhat higher than those that might be expected for the efficient coupling of compressional energy to shear Alfvén FLRs. The auroral arcs associated with the FLR had characteristic latitudinal widths $(10 \mathrm{~km})$ of those expected for field-aligned currents in a toroidal FLR. The periodic restructuring and poleward motion (as clearly seen in the MPA data) also give strong evidence for a toroidal FLR. The FLR model described in this paper includes most of the important physical elements, and is able to explain most features of the observations. The model suggests that the FLR observed by FAST existed on stretched field lines in the magnetotail, and magnetic field configurations from the T96 model with stretched field lines can explain the very low frequency compared to frequencies expected in more dipolar configurations. Wave dispersive effects related to thermal electron motion might explain the relation between the amplitudes of the azimuthal magnetic fields, radial electric fields, and the parallel electric current. Finally, a nonlocal electron conductivity model provides the wave dispersion that explains the latitudinal structure of the fields and currents, and also the amplitude of the parallel electric fields, their location, and the energy of precipitating electrons in the ionosphere. 
Acknowledgements. Topical Editor G. Chanteur thanks three referees for their help in evaluating this paper.

\section{References}

Antonsen, T. M. and Lane, B.: Kinetic calculations for low frequency instabilities in inhomogeneous plasmas, Phys. Fluids, 23, 1205, 1980.

Atkinson, G.: Mechanism by which merging at X-lines causes discrete auroral arcs, J. Geophys. Res., 97, 1337, 1992.

Borovsky, J. E.: Auroral arc thickness as predicted by various theories, J. Geophys. Res., 98, 6101, 1993.

Carlson, C. W., Pfaff, R. F., and Watson, J. C.: The Fast Auroral Snapshot (FAST) mission, Geophys. Res. Lett., 25, 2013, 1998.

Chan, A. A., Xia, M., and Chen, L.: Anisotropic Alfvén-ballooning modes in Earth's magnetosphere, J. Geophys. Res., 99, 17351 , 1994.

Chiu, Y. T. and Schulz, M.: Self-consistent particle and parallel electrostatic field distributions in the magnetosphericionospheric auroral regions, J. Geophys. Res., 83, 629, 1978.

Fenrich, F. R., Samson, J. C., Sofko, G., and Greenwald, R. A.: ULF high and low-m field line resonances observed with SuperDARN, J. Geophys. Res., 100, 535, 1995.

Fridman, M. and Lemaire, L.: Relationship between auroral electron fluxes and field aligned potential difference, J. Geophys. Res., 85, 644, 1980.

Frycz, P., Rankin, R., Samson, J. C., and Tikhonchuk, V. T.: Nonlinear field line resonances: Dispersive effects, Phys. Plasmas, 5, $3565,1998$.

Goertz, C. K.: Kinetic Alfvén waves on auroral field lines, Planet. Space Sci., 32, 1387, 1984.

Kivelson, M. G. and Southwood, D. J.: Coupling of global magnetospheric MHD eigenmodes to field line resonances, J. Geophys. Res., 91, 4345-4351, 1986.

Knight, S.: Parallel electric fields, Planet. Space Sci., 21, 741, 1973.

Lee, D.-H., Lysak, R. L., and Song, Y.: Generation of field-aligned current in the near-Earth magnetotail, Geophys. Res. Lett., 28, 1883, 2001.

Liu, W. W., Xu, B.-L., Samson, J. C., and Rostoker, G.: Theory and observation of auroral substorms: A magnetohydrodynamic approach, J. Geophys. Res., 99, 21 291, 1994.

Lotko, W., Streltsov, A. V., and Carlson, C. W.: Discrete auroral arcs, electrostatic shocks and suprathermal electrons powered by dispersive, anomalously resistive field line resonance, Geophys. Res. Lett., 25, 4449, 1998.

Lyons, L. R. and Samson, J. C.: Formation of the stable auroral arc that breaks-up at substorm onset, Geophys. Res. Lett., 19, 2171, 1992.

Mitchell, D. G., Engebretson, M. J., Williams, D. J., Cattell, C. A., and Lundin, R.: Pc5 pulsations in the outer dawn magnetosphere seen by ISEE land 2, J. Geophys. Res., 95, 967, 1990.
Newell, P. T., Sergeev, V. A., Bikkuzina, G. R., and Wing, S.: Characterizing the state of the magnetosphere: Testing the ion precipitation maxima latitude (b2I) and the ion isotropy boundary, J. Geophys. Res., 103, 4739, 1998.

Rankin, R., Samson, J. C., Tikhonchuk, V. T., and Voronkov, I.: Auroral density fluctuations on dispersive field line resonances, J. Geophys. Res., 104, 4399, 1999a.

Rankin, R., Samson, J. C., and Tikhonchuk, V. T.: Discrete auroral arcs and nonlinear dispersive field line resonances, Geophys. Res. Lett., 26, 663, 1999b.

Rankin, R., Samson, J. C., and Tikhonchuk, V. T.: Parallel electric fields in dispersive shear Alfvén waves in the dipolar magnetosphere, Geophys. Res. Lett., 26, 3601, 1999c.

Rankin, R., Fenrich, F., and Tikhonchuk, V. T.: Shear Alfvén waves on stretched magnetic field lines near midnight in Earth's magnetosphere, Geophys. Res. Lett., 27, 3265, 2000.

Rostoker, G., Samson, J. C., Creutzberg, T., Hughes, T. J., McDiarmid, D. R., McNamara, A. G., Vallance Jones, A., Wallis, D. D., and Cogger, L. L.: CANOPUS - A ground based instrument array for remote sensing in the high latitude ionosphere during ISTP/GGS program, Space Sci. Rev., 71, 743, 1994.

Ruohoniemi, J. M., Greenwald, R. A., Baker, K. B., and Samson, J. C.: HF radar observations of Pc5 field line resonances in the midnight/early morning MLT sector, J. Geophys. Res., 96, 15697, 1991.

Samson, J. C., Harrold, B. G., Ruohoniemi, J. M., Greenwald, R. A., Baker, K. B., and Walker, A. D. M.: Field line resonances associated with MHD waveguides in the magnetosphere, Geophys. Res. Lett., 19, 441, 1992a.

Samson, J. C., Lyons, L. R., Newell, P. T., Creutzberg, F., and Xu, B.: Proton aurora and substorm intensifications, Geophys. Res. Lett., 19, 2167, 1992b.

Samson, J. C.: Mapping substorm intensifications from the ionosphere to the magnetosphere, in: Proc. of Int. Conf. on Substorms 2, (Eds) Kan, J. R., Craven, J. D., and Akasofu, S.-I., University of Alaska, Fairbanks, 237, 1994.

Samson, J. C., Cogger, L. L., and Pao, Q.: Observations of field line resonances, auroral arcs, and auroral vortex structures, J. Geophys. Res., 101, 17 373, 1996.

Sergeev, V. A., Malkov, M. V., and Marsula, V.: Testing of the isotropic boundary algorithm method to evaluate the magnetic field configuration in the tail, J. Geophys. Res., 98, 7609, 1993.

Tikhonchuk, V. T. and Rankin, R.: Electron kinetic effects in standing shear Alfvén waves in the dipolar magnetosphere, Phys. Plasmas 7, 2630, 2000.

Tsyganenko, N. A.: Effects of the solar wind conditions on the global magnetospheric configuration as deduced from data-based models, in: Proc. of Third Int. Conf. on Substorms (ICS-3), Eur. Space Agency Publ., ESA SP-389, 181, 1996.

Waters, C. L., Samson, J. C., and Donovan, E. F.: Variation of plasmathrough density derived from magnetospheric field line resonances, J. Geophys. Res., 101, 24 737, 1996. 